\title{
The pinched Veronese is Koszul
}

\author{
Giulio Caviglia
}

Received: 12 October 2008 / Accepted: 2 March 2009 / Published online: 20 March 2009

(C) Springer Science+Business Media, LLC 2009

\begin{abstract}
In this paper we prove that the coordinate ring of the pinched Veronese, $k\left[X^{3}, X^{2} Y, X Y^{2}, Y^{3}, X^{2} Z, Y^{2} Z, X Z^{2}, Y Z^{2}, Z^{3}\right]$, is Koszul. The result is obtained by combining the use of a flat deformation induced by a distinguished weight together with a generalization of the notion of Koszul filtrations.
\end{abstract}

Keywords Koszul algebras · Pinched Veronese $\cdot$ Koszul filtration

\section{Introduction}

A standard graded $k$-algebra $R$ is said to be Koszul if its residue field $k$ has a linear free resolution as an $R$-module. It is a well known fact that a Koszul algebra $R$ has to be quadratic in the sense that there exists a presentation $R \cong k\left[X_{1}, \ldots, X_{n}\right] / I$ where $I$ is generated by homogeneous forms of degree two. The converse does not hold in general; the first counterexample was found by $\mathrm{C}$. Lech and consists of $k\left[X_{1}, \ldots, X_{4}\right] / I$ where $I$ is generated by five generic quadratic forms. On the other hand, examples of Koszul algebra are those defined by quadratic Gröbner bases or by quadratic complete intersections. Both conditions are however not necessary for an algebra to be Koszul. A good survey on Koszul algebras can be found in [10].

Many classical varieties, like Grassmannians, Veronese and Segre varieties are not only Koszul but they are presented by a quadratic Gröbner basis in their natural presentations. Furthermore, given $R$ any commutative graded $k$-algebra the Veronese subalgebra $R^{(d)}$ has a quadratic Gröbner basis for $d \gg 0$ [9], and so in particular it is

The author was partially supported by the "Istituto Nazionale di Alta Matematica Francesco Severi", Rome.

G. Caviglia $(\bowtie)$

Mathematics Department, Purdue University, 150 N. University Street, West Lafayette,

IN 47907-2067, USA

e-mail: gcavigli@math.purdue.edu 
Koszul. This result was later generalized to a larger class of algebras not necessarily generated in degree one, see [4]. Sturmfels in [14] has shown that the $k$-algebra called of Veronese type i.e the subring of $S=k\left[x_{1}, \ldots, x_{n}\right]$ generated by the monomials $\left\{x_{1}^{i_{1}} \cdots x_{n}^{i_{n}} \mid i_{1}+\cdots+i_{n}=d, 0 \leq i_{1} \leq s_{1}, \ldots, 0 \leq i_{n} \leq s_{n}\right\}$ has a Gröbner basis, in a certain ordering, which is not only quadratic but also square-free. Note that when $s_{1}=\cdots=s_{n}=d$ we get $S^{(d)}$. Further generalizations have been done by Blum in [2].

An important and open question is whether any quadratic toric variety with an isolated singularity is Koszul. The pinched Veronese, i.e. the $k$-algebra, where $k$ is a field, defined as $R=k\left[X^{3}, X^{2} Y, X Y^{2}, Y^{3}, X^{2} Z, Y^{2} Z, X Z^{2}, Y Z^{2}, Z^{3}\right]$, has been for a long time the first and the simplest case of the previous question where the answer was unknown. Indeed it is the coordinate ring of a generic projection to $\mathbb{P}^{8}$ of the Veronese embedding $\mathbb{P}^{2} \rightarrow \mathbb{P}^{9}$ of degree 3 and, being so, it has isolated singularity.

The problem about the Koszulness of the pinched Veronese was raised by Sturmfels in the 1993 in a conversation with Peeva, and after that has been circulating as a concrete example to test the efficiency of the new theorems and techniques concerning Koszul algebras.

In Section 3 of this paper we are going to prove that the pinched Veronese is Koszul, i.e.

Theorem 3.1 The algebra $R=k\left[X^{3}, X^{2} Y, X Y^{2}, Y^{3}, X^{2} Z, Y^{2} Z, X Z^{2}, Y Z^{2}, Z^{3}\right]$, where $k$ is a field, is Koszul.

\section{Initial ideal with respect to a weight and Koszul filtrations for modules}

It is well known that a graded algebra $k\left[X_{1} \ldots, X_{n}\right] / I$ with $I \subseteq\left(X_{1}, \ldots, X_{n}\right)^{2}$ is Koszul if, for a certain term order, it can be generated by a Gröbner basis of quadratic forms. If $I$ is monomial the converse holds [3] , consequently, if for a certain term order in $(I)$ defines a Koszul algebra, so does $I$. It is known that the same result is still true considering, instead of a term order, a weight function $w$ given by a vector $\left(w_{1}, \ldots, w_{n}\right)$ of positive integers and replacing in $(I)$ by the initial ideal $\operatorname{in}_{w}(I)$ (not necessary monomial) of $I$ with respect to $w$. The proof of this fact is based on the idea of flat families and we refer for notations and generalities concerning them to [8] 15.8 .

Given a weight function $w=\left(w_{1}, \ldots, w_{n}\right)$ from $\mathbb{Z}^{n}$ to $\mathbb{Z}$ we can think about it as a function defined on monomials of $R$; moreover given $f \in R$ we use $\operatorname{in}_{w}(f)$ for the sum of all the terms of $f$ that are maximal with respect to $w$. Given an ideal $I$ we write $\operatorname{in}_{w}(I)$ for the ideal generated by $\operatorname{in}_{w}(f)$ for all $f \in I$. Let $S=R[T]$ be a polynomial ring in one variable over $R$; for any $f \in R$ we define $\tilde{f}$ in the following way: we can write $f=\sum u_{i} m_{i}$ where $m_{i}$ are distinct monomials and $0 \neq u_{i} \in k$. Let $a=\max w\left(m_{i}\right)$, and set

$$
\tilde{f}=T^{a} f\left(T^{-w_{1}} X_{1}, \ldots, T^{-w_{n}} X_{n}\right) .
$$


Note that $\tilde{f}$ can be written as $\operatorname{in}_{\omega}(f)+g T$ where $g$ belongs to $S$. For any ideal $I$ of $R$ define $\tilde{I}$ to be the ideal of $S$ generated by the elements $\tilde{f}$ for all $f \in I$. Setting $\operatorname{deg} X_{i}=\left(1, w_{i}\right)$ and $\operatorname{deg} T=(0,1)$ the algebra $S$ is bigraded and in particular if $I \subset R$ is an homogeneous ideal then $\tilde{I}$ is bihomogeneous. From the definition it follows that $S /((T)+\tilde{I}) \cong R / \operatorname{in}(I)$ and $S /((T-1)+\tilde{I}) \cong R / I$. We claim that $T$ is a non-zerodivisor on $S / \tilde{I}$. Let $T f \in \tilde{I}$ for some $f \in S$. Without loss of generality we can assume $f$ bihomogeneous and moreover specializing at $T=1$ we have $h=$ $f\left(X_{1}, \ldots, X_{n}, 1\right) \in I$, but $f$ is bihomogeneous therefore it holds that $f=\tilde{h} \in \tilde{I}$. From this fact it follows that also $T-1$ is a non-zerodivisor for $S / \tilde{I}$ since it is sum of two non-zerodivisors of different degrees.

The following statement is folklore, but since we couldn't find a proof in the literature we give one here for completeness.

Lemma 2.1 Let $R=k\left[X_{1}, \ldots, X_{n}\right]$. Consider a weight given by a vector of positive integers $w=\left(w_{1}, \ldots, w_{n}\right)$ and homogeneous ideals $I, J, H$ such that $I \subseteq J$ and $I \subseteq H$. Then

$$
\operatorname{dim}_{k} \operatorname{Tor}_{i}^{R / I}(R / J, R / H)_{j} \leq \operatorname{dim}_{k} \operatorname{Tor}_{i}^{R / \operatorname{in}_{w} I}\left(R / \operatorname{in}_{w} J, R / \operatorname{in}_{w} H\right)_{j} .
$$

Proof Consider the ideals $\tilde{I}, \tilde{J}, \tilde{H}$ of $S=R[T]$ defined as above. Let $M_{i}=$ $\operatorname{Tor}_{i}^{S / \tilde{I}}(S / \tilde{J}, S / \tilde{H})$; note that $M_{i}$ is bigraded and we can make it a $\mathbb{Z}$-graded module setting $\left(M_{i}\right)_{j}=\bigoplus_{h \in \mathbb{Z}}\left(M_{i}\right)_{(j, h)}$. Since $\left(M_{i}\right)_{j}$ is a finitely generated module over $k[T]$, the structure theorem for modules over a PID applies and we obtain the isomorphism $\left(M_{i}\right)_{j} \cong k[T]^{a_{i j}} \bigoplus A_{i j}$ where $A_{i j}$ is the torsion submodule. Moreover since $A_{i j}$ has to be homogeneous the structure theorem gives $A_{i j} \cong \bigoplus_{h=1}^{b_{i j}} k[T] /\left(T^{d_{h}}\right)$. Set $l_{1}=T$ and $l_{2}=T-1$ and consider the following exact sequence

$$
0 \rightarrow S / \tilde{H} \stackrel{\cdot l_{r}}{\rightarrow} S / \tilde{H} \rightarrow S /\left(\left(l_{r}\right)+\tilde{H}\right) \rightarrow 0
$$

for $r=1,2$. All the modules appearing in (2.1.1) are over $S / \tilde{I}$ and the multiplication by $l_{i}$ is a zero degree map with respect to the $\mathbb{Z}$-grading. Tensoring with $S / \tilde{J}$ and passing to the long exact sequence of homologies we have

$$
0 \rightarrow M_{i} / l_{r} M_{i} \rightarrow \operatorname{Tor}_{i}^{S / \tilde{I}}\left(S / \tilde{J}, S /\left(\left(l_{r}\right)+\tilde{H}\right)\right) \rightarrow \operatorname{ker}\left(M_{i-1} \stackrel{\cdot l_{r}}{\rightarrow} M_{i-1}\right) \rightarrow 0
$$

Since $l_{i}$ is a regular element for $S / \tilde{I}$ and $S / \tilde{J}$, the middle term is isomorphic to $\operatorname{Tor}_{i}^{S /\left(\left(l_{r}\right)+\tilde{I}\right)}\left(S /\left(\left(l_{r}\right)+\tilde{J}\right), S /\left(\left(l_{r}\right)+\tilde{H}\right)\right)$ (see [13] Lemma 2 page 140) which is $\operatorname{Tor}_{i}^{R / \text { in }_{w} I}\left(R / \operatorname{in}_{w} J, R / \operatorname{in}_{w} H\right)$ when $r=1$ and is $\operatorname{Tor}_{i}^{R / I}(R / J, R / H)$ for $r=2$.

Therefore taking the graded component of degree $j$ in (2.1.2) we obtain:

$$
\begin{gathered}
\operatorname{dim}_{k} \operatorname{Tor}_{i}^{R / \operatorname{in}_{w} I}\left(R / \operatorname{in}_{w} J, R / \operatorname{in}_{w} H\right)_{j}=a_{i j}+b_{i j}+b_{(i-1) j}, \\
\operatorname{dim}_{k} \operatorname{Tor}_{i}^{R / I}(R / J, R / H)_{j}=a_{i j} .
\end{gathered}
$$

The Lemma follows by comparing (2.1.3) and (2.1.4). 
Corollary 2.2 Let $w$ be a weight function and I be a homogeneous ideal of $R=$ $k\left[X_{1}, \ldots, X_{n}\right]$ such that $R / \operatorname{in}_{w}(I)$ is Koszul. Then $R / I$ is Koszul.

Proof Since $R / \operatorname{in}_{w} I$ is Koszul, $\operatorname{dim}_{k} \operatorname{Tor}_{i}^{R / \operatorname{in}_{w} I}(k, k)_{j}=0$ for any $i \neq j$. Applying Lemma 2.1 with $J=H=\left(X_{1}, \ldots, X_{n}\right)$, we see that $\operatorname{Tor}_{i}^{R / I}(k, k)_{j}=0$ for any $i \neq j$.

We recall the definition of a Koszul filtration introduced by A. Conca, N.V. Trung and G. Valla in [7], see also [12] for related results.

Definition 2.3 Let $R$ be a standard graded $k$-algebra. A family $\mathbf{F}$ of ideals of $R$ is said to be a Koszul filtration of $R$ if:

1) Every ideal $I \in \mathbf{F}$ is generated by linear forms.

2) The ideal (0) and the maximal homogeneous ideal $\mathcal{M}$ of $R$ belong to $\mathbf{F}$.

3) For every $I \in \mathbf{F}$ different from (0), there exists $J \in \mathbf{F}$ such that $J \subset I, I / J$ is cyclic and $J: I \in \mathbf{F}$.

In [7] it is proved that all the ideals belonging to such a filtration have a linear free resolution over $R$ and in particular, since the homogeneous maximal ideal is in $\mathbf{F}, R$ will be a Koszul algebra. For the purposes of this paper, we need an extension of this definition to the case of graded modules. In particular we want $\mathbf{F}$ to be a collection of finitely generated graded modules.

Definition 2.4 Let $R$ be a standard graded $k$-algebra. A family $\mathbf{F}$ of finitely generated graded $R$-modules is said to be a Koszul filtration for modules if the following three properties hold:

1) Every nonzero module $M \in \mathbf{F}$ is generated by its nonzero component of lowest degree, say $s_{M}$.

2) The zero module belongs to $\mathbf{F}$.

3) For every $M \in \mathbf{F}$ different from the zero module there exists $N \subsetneq M, N \in \mathbf{F}$ with $N=0$ or $s_{M}=s_{N}$, such that either $M / N$ has a linear free resolution (i.e $\operatorname{Tor}_{i}^{R}(M / N, k)_{j}=0$ for all $\left.j \neq i+s_{M}\right)$ or the module of first syzygies $\Omega_{1}^{R}(M / N)$ of $M / N$ is generated in degree $s_{M}+1$ and $\Omega_{1}^{R}(M / N)(1) \in \mathbf{F}$.

The next proposition shows that all the elements in $\mathbf{F}$ have a linear free resolution over $R$. In particular, because of this fact, the problem of proving that some module $M$ has a linear free resolution over $R$ can be approached by trying to construct a Koszul filtration containing $M$. This way of proceeding is very useful in the next section where the Koszul filtration for modules plays an important role in proving that the pinched Veronese is Koszul.

Proposition 2.5 Let $R$ be a standard graded $k$-algebra and $\mathbf{F}$ a Koszul filtration as defined in (2.4). Then every $M \in \mathbf{F}$ has a linear free resolution over $R$.

The proof of this result is essentially the same as the one for the case of a Koszul filtration (see [7] Prop. 1.2). 
Proof We need to show that for every $M \in \mathbf{F}$ we have $\operatorname{Tor}_{i}^{R}(M, k)_{j}=0$ for all $j \neq i+s_{M}$. We argue by induction on the index $i$. If $i=0$ the assertion is clearly true; fix an integer $i>0$. If $M$ is the zero module it has obviously a linear resolution, therefore we can assume that $M$ has a positive minimum number of generators $\mu(M)$. Inducting on $\mu(M)$ we can assume that $\operatorname{Tor}_{i}^{R}(N, k)_{j}=0$ whenever $j \neq i+s_{N}, N \in \mathbf{F}$ and $\mu(N)<\mu(M)$. The third property in Definition 2.4 implies that $M$ has a submodule $N \subsetneq M$ with $s_{M}=s_{N}$ and in particular with $\mu(N)<\mu(M)$.

The short exact sequence

$$
0 \longrightarrow N \longrightarrow M \longrightarrow M / N \longrightarrow 0
$$

gives the exact sequence

$$
\operatorname{Tor}_{i}^{R}(N, k)_{j} \longrightarrow \operatorname{Tor}_{i}^{R}(M, k)_{j} \longrightarrow \operatorname{Tor}_{i}^{R}(M / N, k)_{j}
$$

From the third property in Definition 2.4 either $M / N$ has a linear resolution, so in particular $\operatorname{Tor}_{i}^{R}(M / N, k)_{j}=0$ for $j \neq i+s_{M}$, or $\Omega_{1}^{R}(M / N)(1) \in \mathbf{F}$ and is generated in degree $s_{M}$. The last term in (2.5.1) is isomorphic to $\operatorname{Tor}_{i-1}^{R}\left(\Omega_{1}^{R}(M / N)(1), k\right)_{j-1}$. Since the inductive hypothesis on the index of the Tor applies we deduce that $\operatorname{Tor}_{i}^{R}(M / N, k)_{j}=\operatorname{Tor}_{i-1}^{R}\left(\Omega_{1}^{R}(M / N)(1), k\right)_{j-1}=0$ when $j \neq i+s_{M}$. On the other hand the induction on the minimum number of generators yields $\operatorname{Tor}_{i}^{R}(N, k)_{j}=0$ for $j \neq s_{M}+i$ and therefore the middle term in (2.5.1) vanishes when $j \neq s_{M}+i$.

Remark 2.6 We consider, in the third property in Definition 2.4, the fact of having a linear free resolution over $R$ as a possible condition for an element $M$ in $\mathbf{F}$. This is not really essential: indeed if we already know that $M$ has a linear free resolution over $R$ we could add to $\mathbf{F}$ all the modules of syzygies of $M$ filtering them, trivially, with 0 . We decide, for the sake of convenience, to try to keep the family $\mathbf{F}$ as small as possible. On the other hand, if we only leave the second part of condition number 3), it is reasonable to ask, given a module $M$ with a linear free resolution over $R$, if there always exists a finite family $\mathbf{F}$ containing $M$.

Remark 2.7 It is easy to see that the notion of Koszul filtration of [7] is a special case of the notion of Koszul filtration for modules. In fact if $J \subset I$ are ideals generated by linear forms (as in Definition 2.3) with $I / J$ cyclic, then $J: I \cong \Omega_{1}^{R}(I / J)(1)$.

Remark 2.8 Our definition of Koszul filtration for modules covers also the definition of module with linear quotients introduced by Conca and Herzog in [5] in order to study the linearity of the free resolution of certain modules over a polynomial ring.

\section{The pinched Veronese}

In this section we prove that the coordinate ring $R$ of the pinched Veronese variety is Koszul. The proof is structured in three different steps. First of all we can consider a presentation for $R$ and write it as $R=S / I$ where $I$ is a homogeneous ideal generated by quadrics and $S$ is a polynomial ring. 
The first step consists in taking the initial ideal of $I$ with respect to a carefully chosen weight $\omega$. By Corollary 2.2, it is then sufficient to show that $S / \operatorname{in}_{\omega}(I)$ is Koszul. The choice of $\omega$ represents a crucial part of the argument. On one hand it is possible to verify, for instance by using the software system CaTS [1], that in the given system of coordinates the ideal $I$ does not admit a quadratic Gröbner basis. A weight which is too general will play the role of a term order, bringing in the initial ideal some minimal generator of degree higher than two, and therefore the ring defined by such an initial ideal cannot be Koszul. On the other hand by taking, for example, the trivial weight $\omega=(1, \ldots, 1)$ we get $\operatorname{in}_{\omega}(I)=I$ and we do not make any simplification. The $\omega$ we use in the proof allows us to study instead of a binomial ideal, an ideal generated by several quadratic monomials and only five quadratic binomials. After an extensive search made with the computer algebra system MACAULAY2 [11] we were unable to find any weight that would give an initial ideal with fewer than five quadratic binomials and would preserve at the same time the linearity of the first few steps of the resolution of $k$ over the corresponding quotient ring.

The second reduction consists in writing $\operatorname{in}_{\omega}(I)$ as the sum two ideals: $U$ generated by the monomial part of $\operatorname{in}_{\omega}(I)$ plus a distinguished binomial of $\operatorname{in}_{\omega}(I)$ and the ideal $\left(Q_{1}, \ldots, Q_{4}\right)$ given by the remaining four binomials of $\operatorname{in}_{\omega}(I)$. The ideal $U$ is generated by a Gröbner basis of quadrics, so $S / U$ is Koszul. We need the following fact, which is part of Lemma 6.6 of [6]:

Fact 1 Let $T$ be a Koszul algebra let $Q \subset T$ be a quadratic ideal with a linear free resolution over $T$. Then $T / Q$ is Koszul.

Using this fact it is enough to show that the class $\left(q_{1}, \ldots, q_{4}\right)$ of $\left(Q_{1}, \ldots, Q_{4}\right)$ in $S / U$ has a linear free resolution over $S / U$. Note that among all the possible five binomials, the distinguished one we pick is the only one giving at the same time the Koszulness of $S / U$ and the linearity of the ideal given by the other four. It is maybe possible to show that the whole binomial part has a linear resolution over $S$ modulo the monomial one, but for this purpose the amount of calculations required seems much higher.

The last part of the proof consists in showing the linearity of the free resolution of $\left(q_{1}, \ldots, q_{4}\right)$ over $S / U$ via the construction of a Koszul filtration containing $\left(q_{1}, \ldots, q_{4}\right)$.

Theorem 3.1 The algebra $R=k\left[X^{3}, X^{2} Y, X Y^{2}, Y^{3}, X^{2} Z, Y^{2} Z, X Z^{2}, Y Z^{2}, Z^{3}\right]$, where $k$ is field, is Koszul.

Proof Since $R$ contains all monomials in $X, Y, Z$ of degree 9 its Hilbert function is $H_{R}(0)=1, H_{R}(1)=9$ and $H_{R}(n)=\left(\begin{array}{c}3 n+2 \\ 2\end{array}\right)$ for $n \geq 2$. The Hilbert polynomial of $R$ is given by $\left(\begin{array}{c}3 n+2 \\ 2\end{array}\right)$ and the Krull dimension of $R$ is 3 . One computes that the Hilbert series of $R$ is given by:

$$
H_{R}(Z)=\frac{Z^{4}-3 Z^{3}+4 Z^{2}+6 Z+1}{(1-Z)^{3}} .
$$


Consider a presentation $S / \operatorname{ker} \phi \cong R$ where $S=k\left[X_{1}, \ldots, X_{9}\right]$ and $\phi$ is the homomorphism from $S$ to $R$ defined by sending $X_{i}$ to the $i^{\text {th }}$ monomial of $R$ in $\left(X^{3}, X^{2} Y, X Y^{2}, Y^{3}, X^{2} Z, Y^{2} Z, X Z^{2}, Y Z^{2}, Z^{3}\right)$. Let $I$ be the ideal defined as

$$
\begin{aligned}
I= & \left(X_{8}^{2}-X_{6} X_{9}, X_{6} X_{8}-X_{4} X_{9}, X_{5} X_{8}-X_{2} X_{9}, X_{7}^{2}-X_{5} X_{9},\right. \\
& X_{6} X_{7}-X_{3} X_{9}, X_{5} X_{7}-X_{1} X_{9}, X_{4} X_{7}-X_{3} X_{8}, X_{3} X_{7}-X_{2} X_{8}, \\
& X_{2} X_{7}-X_{1} X_{8}, X_{6}^{2}-X_{4} X_{8}, X_{5} X_{6}-X_{2} X_{8}, X_{5}^{2}-X_{1} X_{7}, X_{4} X_{5}-X_{2} X_{6}, \\
& \left.X_{3} X_{5}-X_{1} X_{6}, X_{3}^{2}-X_{2} X_{4}, X_{2} X_{3}-X_{1} X_{4}, X_{2}^{2}-X_{1} X_{3}\right) .
\end{aligned}
$$

It is immediate to see that $I \subseteq \operatorname{ker} \phi$. On the other hand also the opposite inclusion holds, in fact it is sufficient to check that $R$ and $S / I$ have the same Hilbert function. We will prove this below.

Consider the weight function $\omega$ from $\mathbb{Z}^{9}$ to $\mathbb{Z}$ given by $(3,3,1,3,3,3,2,3,3)$ and take its natural extension to the monomials of $S$. Let $J$ be the ideal generated by the initial forms with respect to $\omega$ of the generators of $I$ given previously. We have

$$
\begin{aligned}
J= & \left(X_{8}^{2}-X_{6} X_{9}, X_{6} X_{8}-X_{4} X_{9}, X_{5} X_{8}-X_{2} X_{9}, X_{5} X_{9}, X_{6} X_{7},\right. \\
& X_{1} X_{9}, X_{4} X_{7}, X_{2} X_{8}, X_{1} X_{8}, X_{6}^{2}-X_{4} X_{8}, X_{5} X_{6}, X_{5}^{2}, \\
& \left.X_{4} X_{5}-X_{2} X_{6}, X_{1} X_{6}, X_{2} X_{4}, X_{1} X_{4}, X_{2}^{2}\right)
\end{aligned}
$$

We claim that $J=\operatorname{in}_{\omega} I$. One inclusion is clear and to prove the other is enough to show, as stated previously, that $R / J$ and $R / I$ have the same Hilbert function. Consider the degrevlex order $\sigma$ on the monomials of $S$. Note first that $X_{2} X_{9}^{2}$ and $X_{2} X_{6} X_{9}$ belong to $J$ since $X_{2} X_{9}^{2}=\left(X_{5} X_{9}\right) X_{8}-\left(X_{5} X_{8}-X_{2} X_{9}\right) X_{9}$ and $X_{2} X_{6} X_{9}=$ $\left(X_{2} X_{8}\right) X_{8}-\left(X_{8}^{2}-X_{6} X_{9}\right) X_{2}$, therefore the following ideal

$$
\begin{aligned}
H= & \left(X_{5} X_{9}, X_{1} X_{9}, X_{8}^{2}, X_{6} X_{8}, X_{5} X_{8}, X_{2} X_{8}, X_{1} X_{8}, X_{6} X_{7}, X_{4} X_{7}, X_{6}^{2},\right. \\
& \left.X_{5} X_{6}, X_{1} X_{6}, X_{5}^{2}, X_{4} X_{5}, X_{2} X_{4}, X_{1} X_{4}, X_{2}^{2}, X_{2} X_{9}^{2}, X_{2} X_{6} X_{9}\right)
\end{aligned}
$$

is contained in $\operatorname{in}_{\sigma} J$. The Hilbert series of $S / H$ is easy to compute and it is $H_{S / H}(Z)=\frac{Z^{4}-3 Z^{3}+4 Z^{2}+6 Z+1}{(1-Z)^{3}}$. Coefficient-wise we have:

$$
H_{S / \operatorname{ker} \phi}(Z) \leq H_{S / I}(Z)=H_{S / \operatorname{in}_{\omega} I}(Z) \leq H_{S / J}(Z)=H_{S / \operatorname{in}_{\sigma} J}(Z) \leq H_{S / H}(Z) .
$$

The first and the last term agree, thus all the previous inequalities are in fact equalities, and in particular it follows that $\operatorname{ker} \phi=I, \operatorname{in}_{\omega} I=J$ and $\operatorname{in}_{\sigma} J=H$.

Applying Corollary 2.2 to $S / I$ and $\omega$, in order to finish the proof of the theorem it is enough to show the following

\section{Claim 1 The k-algebra S/J is Koszul.}

Proof of the claim We can write $J$ as a sum of two ideals: one generated by all the quadratic monomials of $J$ together with the quadratic binomial $X_{6} X_{8}-X_{4} X_{9}$, 
namely

$$
\begin{aligned}
U= & \left(X_{5} X_{9}, X_{1} X_{9}, X_{2} X_{8}, X_{1} X_{8}, X_{6} X_{7}, X_{4} X_{7}, X_{5} X_{6}, X_{1} X_{6}, X_{5}^{2}, X_{2} X_{4},\right. \\
& \left.X_{1} X_{4}, X_{2}^{2}, X_{6} X_{8}-X_{4} X_{9}\right),
\end{aligned}
$$

and the other one generated by the remaining binomials $Q_{1}=X_{6}^{2}-X_{4} X_{8}, Q_{2}=$ $X_{4} X_{5}-X_{2} X_{6}, Q_{3}=X_{8}^{2}-X_{6} X_{9}$ and $Q_{4}=X_{5} X_{8}-X_{2} X_{9}$. Note first that $U$ is generated by a Gröbner basis of quadrics with respect to the degrevlex order $\sigma$, in fact all the S-pairs we need to check are:

$$
\begin{aligned}
& \left(X_{6} X_{8}-X_{4} X_{9}\right) X_{2}-\left(X_{2} X_{8}\right) X_{6}=-\left(X_{2} X_{4}\right) X_{9}, \\
& \left(X_{6} X_{8}-X_{4} X_{9}\right) X_{1}-\left(X_{1} X_{8}\right) X_{6}=-\left(X_{1} X_{9}\right) X_{4}, \\
& \left(X_{6} X_{8}-X_{4} X_{9}\right) X_{7}-\left(X_{6} X_{7}\right) X_{8}=-\left(X_{4} X_{7}\right) X_{9}, \\
& \left(X_{6} X_{8}-X_{4} X_{9}\right) X_{5}-\left(X_{5} X_{6}\right) X_{8}=-\left(X_{5} X_{9}\right) X_{4}, \\
& \left(X_{6} X_{8}-X_{4} X_{9}\right) X_{1}-\left(X_{1} X_{8}\right) X_{6}=-\left(X_{1} X_{9}\right) X_{4} .
\end{aligned}
$$

One can observe that for any ideal $L=\left(X_{i_{1}}, \ldots, X_{i_{r}}\right)$ generated by variables, the ideal $U+L$ is again generated by a Gröbner basis of at most quadrics. Indeed there are no S-pairs to check other than the ones in (3.1.1). Moreover if $L$ is chosen in a such a way that $X_{6} X_{8}-X_{4} X_{9} \in L$ or $X_{6} X_{8} \notin L$, we obtain that $\operatorname{in}_{\sigma}(U)+L=$ $\operatorname{in}_{\sigma}(U+L)$, in fact the only case in which this very last equality doesn't hold is when $X_{4} X_{9}$ appears in the sum without being in $L$. By Theorem 2.2 of [3] if in $\operatorname{in}_{\sigma}(U)+L=$ $\operatorname{in}_{\sigma}(U+L)$ then not only $S / U$ is Koszul but also the ideal $(L+U) / U$ has a linear free resolution over $S / U$.

Now set $S / U=T$. In the following we will denote by $x_{i}$ the class of $X_{i}$ and by $q_{j}$ the class of $Q_{j}$ in $T$. Since $T$ is Koszul we can use Fact 1 to conclude the proof of Claim 1 by showing that $\left(q_{1}, \ldots, q_{4}\right)$ has a linear free resolution over $T$. We prove this by constructing a Koszul filtration $\mathbf{F}$ over $T$ containing $\left(q_{1}, \ldots, q_{4}\right)(1)$ because this implies, by Proposition 2.5 , that $\left(q_{1}, \ldots, q_{4}\right)(1)$ has a linear free resolution over $T$ and so $\left(q_{1}, \ldots, q_{4}\right)$ does.

It will be useful to include in $\mathbf{F}$ a set of ideals $\mathbf{G}$ for which we already know they have a linear free resolution over $T$. Setting

$$
\begin{aligned}
\mathbf{G}= & \left\{\text { Ideals }\left(x_{i_{1}}, \ldots, x_{i_{r}}\right) \text { of } T \text { such that } X_{6} X_{8}-X_{4} X_{9} \in\left(X_{i_{1}}, \ldots, X_{i_{r}}\right)\right. \\
& \text { or } \left.X_{6} X_{8} \notin\left(X_{i_{1}}, \ldots, X_{i_{r}}\right)\right\},
\end{aligned}
$$

from what we have seen above any ideal in $\mathbf{G}$ has a linear resolution over $T$.

We define $\mathbf{F}$ to be

$$
\begin{gathered}
\mathbf{F}=\mathbf{G} \cup\left\{\left(q_{1}, q_{2}\right)(1),\left(q_{1}, \ldots, q_{4}\right)(1), M_{1}^{1, \ldots, 8}, M_{1}^{1,2,4,6,7,8},\right. \\
\left.M_{2}^{1, \ldots, 8}, M_{2}^{1,2,4,5,7,8}, M_{3}^{1, \ldots, 10}, M_{3}^{1,2,3,5,6,7,8,9,10}\right\} \cup\{0\} .
\end{gathered}
$$


The modules $M_{1}^{1, \ldots, 8}, M_{1}^{1,2,4,6,7,8}, M_{2}^{1, \ldots, 8}, M_{2}^{1,2,4,5,7,8}, M_{3}^{1, \ldots, 10}, M_{3}^{1,2,3,5,6,7,8,9,10}$ are constructed as follows. We consider $T$-homomorphisms defined by matrices:

$$
\begin{aligned}
& M_{1}=\left(\begin{array}{cccccccc}
x_{7} & 0 & x_{5} & x_{1} & x_{2} & 0 & 0 & 0 \\
0 & x_{7} & x_{8} & 0 & x_{6} & x_{5} & x_{2} & x_{1}
\end{array}\right) \\
& M_{2}=\left(\begin{array}{cccccccc}
x_{7} & 0 & -x_{8} & 0 & x_{2} & -x_{5} & x_{1} & 0 \\
0 & x_{7} & x_{6} & x_{5} & 0 & x_{2} & 0 & x_{1}
\end{array}\right) \\
& M_{3}=\left(\begin{array}{cccccccccc}
x_{6} & 0 & x_{5} & -x_{8} & x_{4} & 0 & x_{2} & 0 & x_{1} & 0 \\
0 & x_{6} & 0 & x_{5} & 0 & x_{4} & 0 & x_{2} & 0 & x_{1}
\end{array}\right) \\
& M_{1}: T(-1)^{8} \rightarrow T^{2}, \quad M_{2}: T(-1)^{8} \rightarrow T^{2}, \quad M_{3}: T(-1)^{10} \rightarrow T^{2} .
\end{aligned}
$$

We use now an upper index notation on the matrices to indicate the module generated by the images of the elements of the standard basis corresponding to those indices: for instance $M_{1}^{1,4}$ is the module generated by the images under $M_{1}$ of $(1,0, \ldots, 0)$ and $(0,0,0,1,0, \ldots, 0)$.

We prove that $\mathbf{F}$ is a Koszul filtration for $T$. For what concerns the elements in $\mathbf{G}$ there is nothing to check since $0 \in \mathbf{F}$ and they have a linear free resolution over $T$. For all the other modules $M \in \mathbf{F}$ we exhibit a submodule $N \in \mathbf{F}, N \subsetneq M$, such that $M / N$ has a linear free resolution or $\Omega_{1}(M / N)(1)$ belongs to $\mathbf{F}$. We have the following isomorphisms

$$
\begin{aligned}
\Omega_{1}\left(\left(q_{1}, q_{2}\right)(1)\right)(1) & \cong M_{1}^{1, \ldots, 8} \in \mathbf{F} \\
\Omega_{1}\left(\left(q_{1}, \ldots, q_{4}\right) /\left(q_{1}, q_{2}\right)(1)\right)(1) & \cong M_{3}^{1, \ldots, 10} \in \mathbf{F} \\
\Omega_{1}\left(M_{1}^{1, \ldots, 8} / M_{1}^{1,2,4,6,7,8}\right)(1) & \cong M_{2}^{1, \ldots, 8} \in \mathbf{F} \\
M_{1}^{1,2,4,6,7,8} / M_{1}^{1,4} & \cong\left(x_{7}, x_{5}, x_{2}, x_{1}\right) \in \mathbf{G} \subseteq \mathbf{F} \\
\Omega_{1}\left(M_{2}^{1, \ldots, 8} / M_{2}^{1,2,4,5,7,8}\right)(1) & \cong M_{1}^{1, \ldots, 8} \in \mathbf{F} \\
M_{2}^{1,2,4,5,7,8} / M_{2}^{1,5,7} & \cong\left(x_{7}, x_{5}, x_{1}\right) \in \mathbf{G} \subseteq \mathbf{F} \\
\Omega_{1}\left(M_{3}^{1, \ldots, 10} / M_{3}^{1,2,3,5,6,7,8,9,10}\right)(1) & \cong\left(x_{6}, x_{5}, x_{4}, x_{2}, x_{1}\right) \in \mathbf{G} \subseteq \mathbf{F} \\
M_{3}^{1,2,3,5,6,7,8,9,10} / M_{3}^{1,3,5,7,9} & \cong\left(x_{6}, x_{4}, x_{2}, x_{1}\right) \in \mathbf{G} \subseteq \mathbf{F}
\end{aligned}
$$

where (3.1.2), (3.1.3), (3.1.4) and (3.1.6) have been checked with the help of the computer algebra system MACAULAY2 [11] over the field of rational numbers. In particular by flat extension these isomorphisms work over any field of characteristic zero. On the other hand we performed by hand exactly the same Gröbner basis based computation, suggested by the calculations over $\mathbb{Q}$. Since integer coefficients different from 1 or -1 never appear, those calculations are enough to prove the previous isomorphisms also over any field of positive characteristic.

In (3.1.5) and (3.1.7) the modules $M_{1}^{1,4}$ and $M_{2}^{1,5,7}$ are clearly isomorphic to $\left(x_{7}, x_{4}\right) \in \mathbf{G} \subseteq \mathbf{F}$ and to $\left(x_{7}, x_{2}, x_{1}\right) \in \mathbf{G} \subseteq \mathbf{F}$ respectively. Similarly in (3.1.9) the 
module $M_{3}^{1,3,5,7,9}$ is isomorphic to $\left(x_{6}, x_{5}, x_{4}, x_{2}, x_{1}\right)$ which belongs to $\mathbf{G} \subseteq \mathbf{F}$. This show that $\mathbf{F}$ is a Koszul filtration and, as we said before, by Proposition 2.5 the ideal $\left(q_{1} \ldots, q_{4}\right)(1)$ has a linear free resolution over $T$. Thus the claim is proved and so is the theorem.

Acknowledgements I like to thank Craig Huneke and Aldo Conca for all the discussions and encouragements which have made this paper possible. I also like to thank Luis Garcia-Puente for showing me how to use CaTS and Bernd Sturmfels for his helpful comments on an early draft of this article.

\section{References}

1. Anders, N.J.: CaTS, a software package for computing state polytopes of toric ideals, available from http://www.soopadoopa.dk/anders/cats/cats.html

2. Blum, S.: Subalgebras of bigraded Koszul algebras. J. Algebra 242(2), 795-809 (2001)

3. Bruns, W., Herzog, J., Vetter, U.: Syzygies and walks. In: Simis, A., Trung, N.V., Valla, G. (eds.) ICTP Proceedings 'Commutative Algebra', pp. 36-57. World Scientific, Singapore (1994)

4. Bruns, W., Gubeladze, J., Trung, N.V.: Normal polytopes, triangulations, and Koszul algebras. J. Reine Angew. Math. 485, 123-160 (1997)

5. Conca, A., Herzog, J.: Castelnuovo-Mumford regularity of products of ideals. Preprint

6. Conca, A., Herzog, J., Trung, N.V., Valla, G.: Diagonal subalgebras of a bigraded algebras and embeddings of blow ups of projective spaces. Amer. J. Math. 119, 859-901 (1997)

7. Conca, A., Trung, N.V., Valla, G.: Koszul property for points in projective spaces. Math. Scand. 89, 201-216 (2001)

8. Eisenbud, D.: Commutative Algebra with a View Toward Algebraic Geometry. Springer, New York (1995)

9. Eisenbud, D., Reeves, A., Totaro, B.: Initial ideals, Veronese subrings, and rates of algebras. Adv. Math. 109, 168-187 (1994)

10. Fröberg, R.: Koszul algebras. In: Advances in Commutative Ring Theory (Fez, 1997). Lecture Notes in Pure and Appl. Math., vol. 205, pp. 337-350. Dekker, New York (1999)

11. Grayson, D., Stillman, M.: Macaulay 2: a software system for algebraic geometry and commutative algebra available over the web at http://www.math.uiuc.edu/Macaualy2

12. Herzog, J., Hibi, T., Restuccia, G.: Strongly Koszul algebras. Math. Scand. 86, 161-178 (2000)

13. Matsumura, H.: Commutative Ring Theory, 2nd edn. Cambridge Studies in Advanced Mathematics, vol. 8. Cambridge University Press, Cambridge (1989). Translated from the Japanese by M. Reid

14. Sturmfels, B.: Gröbner Bases and Convex Polytopes. University Lecture Series, vol. 8. American Mathematical Society, Providence (1996), xii+162 pp. 